\title{
Lapurdum
}

Euskal ikerketen aldizkaria | Revue d'études basques |

Revista de estudios vascos | Basque studies review

$8 \mid 2003$

Numéro VIII

\section{Dictionnaire français-occitan (gascon) de Michel Grosclaude, Gilbert Narioo et Patric Guilhemjoan}

\author{
Joan Miquèu Dordeins
}

\section{OpenEdition}

Journals

Édition électronique

URL : http://journals.openedition.org/lapurdum/1110

DOI : 10.4000/lapurdum. 1110

ISSN : 1965-0655

Éditeur

IKER

Édition imprimée

Date de publication : 1 novembre 2003

Pagination : 519-521

ISBN : 9782867813436

ISSN : $1273-3830$

Référence électronique

Joan Miquèu Dordeins, « Dictionnaire français-occitan (gascon) de Michel Grosclaude, Gilbert Narioo et Patric Guilhemjoan », Lapurdum [En ligne], 8 | 2003, mis en ligne le 01 juin 2009, consulté le 21 septembre 2020. URL : http://journals.openedition.org/lapurdum/1110; DOI : https://doi.org/ 10.4000/lapurdum. 1110 


\section{Joan Miquèu Dordeins}

\section{Dictionnaire français-occitan (gascon) de Michel Grosclaude, Gilbert Narioo et Patric Guilhemjoan.}

L'association Per Noste, section béarnaise de l'Institut d'Estudis Occitans, vient de publier la première partie de son dictionnaire françaisoccitan. Cet ouvrage qui va de la lettre $\mathrm{A}$ à la lettre $\mathrm{K}$ compte 40000 mots d'entrée en français. Il vient en remplacement d'un petit dictionnaire de 7000 mots qui aura été bien utile à bon nombre de débutants.

L'objectif de ce nouvel ouvrage était de donner à la langue occitane dans sa forme gasconne un outil dans lequel soient répertoriés tous les mots et toutes les expressions que doit posséder une langue moderne. En un mot un dictionnaire dans lequel on trouve ce que l'on cherche et non pas ce que l'on connaît déjà.

Ce dictionnaire est l'aboutissement de vingt années de travail. Dans les années 70 une équipe d'une cinquantaine de personnes s'est lancée dans l'élaboration d'un dictionnaire français-occitan. Les fiches manuscrites (car rédigées avant l'ère de l'informatique) sont restées, pour diverses raisons, quelques années en attente avant d'être reprises en 1996 par Michel Grosclaude et Gilbert Narioo auxquels s'est adjoint Patric Guilhemjoan. Michel Grosclaude, décédé en 2002, ne verra pas l'achèvement de son œuvre.

Ce dictionnaire est l'équivalent occitan du Petit Robert ouvrage de référence de la langue française qu'il suit mot à mot. Pour l'élaborer les auteurs ont eu principalement recours à divers ouvrages occitans anciens ou récents: les dictionnaires de Palay, Lespy et Raymond, Alibert, Taupiac, Laux, Coupier; mais également l'encyclopédie catalane et le dictionnaire francès-catalan de Castellanos i Lorenç (la langue occitane est très proche de la langue catalane) ainsi que l'atlas linguistique de la Gascogne. 
L'aire linguistique occitane compte quatre grands ensembles dialectaux : le gascon, le languedocien, le nord-occitan et le provençal. $\mathrm{Ce}$ dictionnaire est un dictionnaire occitan dans sa variété gasconne. La Gascogne linguistique s'étend sur un triangle dont les côtés sont très approximativement l'océan atlantique, les Pyrénées et la Garonne.

Les auteurs de ce dictionnaire se sont fixé plusieurs orientations générales. La première est la pangasconité de la langue. Il peut servir de Bordeaux à Toulouse en passant par Auch et Tarbes. De nombreuses variantes locales sont données avec précision du lieu d'emploi des mots, soit par la mention d'une région: Armagnac, Gironde, Lomagne...; soit la mention d'une ville: Arcachon, Bayonne, Orthez...

Tout au long de la rédaction, les auteurs ont eu à faire face à plusieurs types de difficultés: les francismes, les néologismes et le vocabulaire technique.

L'occitan est parlé par des gens qui parlent également le français et les deux langues s'influencent mutuellement. Il a fallu épurer la langue. Certains francismes évidents ont été éliminés *fama (femme), d'autres ont été signalés comme tels et suivis du mot proposé en remplacement avec avertissement au lecteur: cahier, *caièr (francisme); quadèrn (mot ancien remis en usage).

Autre difficulté, comment dire "essuie-glace", "ordinateur", "télécommande" ...? Comment dire ces mots nouveaux que toute langue doit posséder? L'occitan, longtemps exclu de l'école, réduit à un ghetto rural, persécuté, n'a pas pu suivre normalement l'évolution des mœurs. La langue s'est appauvrie dans son usage quotidien. Le choix des auteurs pour l'apport de mots nouveaux a été de proposer plutôt que d'imposer.

Pour cela, plusieurs stratégies ont été utilisées. Les auteurs ont remis en usage des mots anciens attestés par des textes tels les fors de Béarn, le for de Henri II, la coutume de la Soule... ben de conquèsta a existé pour exprimer le mot "acquet". Les auteurs ont étendu le sens de certains mots à des acceptions actuelles : lo brasèr, coffre dans lequel on plaçait les cendres du fourneau est devenu le cendrier qui recueille les cendres de cigarettes.

Les auteurs ont enfin fabriqué des mots selon les règles de la langue. Le verbe virar qui signifie, "protéger", a servi à former lo vira- 
vent, le pare-brise. La limite de cet exercice était de proposer des mots qui puissent être compris de tous, immédiatement. L'un des problèmes parmi les plus surprenants a été de traduire le mot forêt. La Gascogne qui comporte le plus vaste massif forestier d'Europe n'a pas de terme générique pour désigner ce type de végétation. Les auteurs ont exhumé le vieux mot seuva au parfum très latin.

Trouver des équivalents à des mots techniques a en revanche été tâche plus aisée. Comme toutes les langues romanes, l'occitan fabrique son vocabulaire technique en empruntant au latin ou au grec. "Constitution", "littérature", "théorème", "oto-rhino-laryngologiste" n'ont posé que peu de problèmes.

L'orthographe adoptée dans ce dictionnaire est celle qui a été mise au point par Louis Alibert voici plus de cinquante ans. Elle n'a subi que peu de modifications depuis. C'est l'orthographe qui est enseignée dans les établissements scolaires et qui est utilisée dans la quasi totalité des publications occitanes. Les auteurs ont scrupuleusement suivi les règles alibertiennes.

Ce dictionnaire s'adresse à tous ceux qui utilisent la langue occitane pour leurs études, dans leur activité professionnelle ou pour leur plaisir. C'est un ouvrage clair, précis, complet.

La sortie de la deuxième partie allant jusqu'à la lettre $\mathrm{Z}$ est prévue pour 2005 . 